\title{
Amine/acid composite Janus nanosheets
}

\author{
Yijiang Liu, Qian Wang, Xiaozhong Qu, Fuxin Liang* and Zhenzhong Yang*
}

\begin{abstract}
Janus materials have witnessed fast development due to their diversified promising performances and practical applications. Compared with their spherical counterparts, Janus nanosheets have gained more concerns for their highly anisotropic shape besides chemistry. Herein, $3.5 \mathrm{~nm}$ ultrathin and flexible Janus nanosheets with carboxyl group terminated onto one side are fabricated by surface sol-gel process of the self-assembled monolayer of an amphiphilic silane onto the template $\mathrm{CaCO}_{3}$ particle firstly. Amine/acid composite Janus nanosheets are further derived from these carboxyl group terminated silica Janus nanosheets by selective conjugation with amine groups onto the other side. The amine/acid composite Janus nanosheets are dually $\mathrm{pH}$ responsive, and well dispersible in aqueous solution at both low and high $\mathrm{pH}$ levels. The nanosheets are aggregated forming multi-layered face-to-back superstructures at intermediate $\mathrm{pH}$ levels. This is originated by the opposite electrostatic interaction between the carboxyl and the amine groups. This approach can be extended to other silanes, and a huge family of Janus nanosheets is expected with tunable composition and performance.
\end{abstract}

\section{INTRODUCTION}

Janus materials, which consist of two different compositions compartmentalized on one object, have been shown to have diverse potential applications, such as solid surfactants, building blocks for superstructures, and self-propellant motors [1-6]. Shape control is important in determining the performances of these materials. Janus nanosheets are particularly interesting because of their highly anisotropic shape besides their asymmetric chemistry. As the rotation of Janus nanosheets at an interface is restricted, the nanosheets appear to form mosaics on the interface that can better stabilize emulsions $[7,8]$. It would thus be useful to develop methods to synthesize Janus nanosheets with tunable thickness and composition.

Polymeric Janus nanosheets have been synthesized by delaminating supramolecular structures formed from alternating layers of block copolymers $[9,10]$. This method requires a sufficiently narrow molecular-weight distribution to ensure uniformity in the supramolecular structure. If this is not the case, the layered structure will coexist with other structures. Janus polymer single crystal nano- plates have been generated by evaporation-induced crystallization of carboxyl-capped poly( $\varepsilon$-caprolactone) at a planar water/pentyl acetate interface [11]. Inorganic Janus nanosheets have been prepared by multistep etching of silicon substrates [12], but the yield is rather low considering that these nanosheets are based on multi-step etching planar substrates. In an effort to increase the yield of the Janus nanosheets, simultaneous atom transfer radical polymerization (ATRP) grafting from the platelets at a Pickering emulsion interface was proposed as a method to synthesize the corresponding Janus nanoplates [13]. It was, however, necessary to properly modify the platelets to form Pickering emulsions. Recently, we proposed a simple way to fabricate silica Janus nanosheets by crushing the corresponding Janus hollow spheres [8]. The Janus hollow spheres were prepared by materialization of the temporal Janus emulsion interface via a self-organized sol-gel process [14]. One side of the silica Janus nanosheets was terminated with amine groups. Then, starting from this side, other polymers were preferentially grown by ATRP to give polymer-silica composite Janus nanosheets. Additional characteristics, such as thermal or $\mathrm{pH}$ responsivity, could also be introduced [15]. However, it was difficult to obtain Janus nanosheets with molecular-scale thickness at the dynamic emulsion interface. A very thin amine/thiol Janus aromatic nanomembrane has been synthesized by electron-irradiation-assisted crosslinking of a monomolecular film on a gold surface or transmission electron microscope (TEM) grid [16]. A Janus graphene monolayer has been synthesized by polymer-mediated transfer-assisted selective grafting of halogen and aryl/oxygen functional groups onto each side of the monolayer [17]. Although they are very thin, these monolayers lack a responsive performance. In addition, the yield is rather low. We have recently developed a method to synthesize flexible Janus nanosheets using a sol-gel process involving a silane self-assembled monolayer (SAM) of (3-butyldianhydride mercaptopropyl)trimethoxysilane (BDMPS) supported on a calcium carbonate $\left(\mathrm{CaCO}_{3}\right)$ particle surface [18]. The nanosheets have molecular-level thickness. After selective modification, one side of the exposed surface has alkyl-terminated groups, whereas the acid group is preserved on the other side. 
Herein, we further extend this approach to synthesize an amine/acid composite $\mathrm{pH}$-responsive Janus nanosheet by conjugation of amine groups on the opposite side of the surface (Scheme 1). A self-assembled monolayer of BDMPS forms with the acid anhydride group attached to the $\mathrm{CaCO}_{3}$ particle surface by a coordination interaction, with the hydrophobic tail group directed towards the external oil phase. The silica Janus nanomembrane is generated through a sol-gel process supported by the particle surface. The hydrophilic side of the surface is synchronously protected, but the exterior silica surface can be selectively modified with silanes, such as (3-aminopropyl)trimethoxysilane. As a result, the amine group is conjugated on the exterior surface. After acid etching of the $\mathrm{CaCO}_{3}$ particle under ultrasonication, the Janus nanomembrane is broken into Janus nanosheets. At the same time, the acid anhydride group is hydrolyzed into a carboxyl group and the amine/acid composite Janus nanosheets are formed. These nanosheets are $\mathrm{pH}$ responsive and their aggregation/dispersion in aqueous solution is $\mathrm{pH}$ triggered.

\section{EXPERIMENTAL SECTION}

\section{Materials}

(3-mercaptopropyl)trimethoxysilane and (3-aminopropyl) trimethoxysilane were purchased from Acros. Maleic anhydride and azobisisobutyronitrile (AIBN) were purchased from Sinopharm Chemical Reagent Beijing. BDMPS was synthesized following the previously reported procedure [18]. Toluene was dried by refluxing with sodium and benzophenone, followed by distillation. Dimethylformamide (DMF) was dried and distilled under reduced pressure. $\mathrm{CaCO}_{3}$ was purchased from Sinopharm Chemical Reagent (Shanghai, China). All reagents were used as received unless otherwise stated.

\section{Characterization}

The morphology of the samples was characterized by scanning electron microscopy (SEM) at $15 \mathrm{kV}$ using a Hitachi S-4800 scanning electron microscope equipped with an energy-dispersive X-ray (EDX) analyzer. The samples for SEM observation were prepared by vacuum sputtering with Pt after ambient drying. Fourier transform infrared (FTIR) spectroscopy was performed by scanning $\mathrm{KBr}$ pressed pellets of the sample 32 times using a Bruker EQUINOX 55 spectrometer. Atomic force microscopy (AFM) images were recorded under ambient conditions using a Digital Instrument Multimode Nanoscope IIIA operating in a tapping mode. A Zeta Sizer 3000 HS (Malvern Instruments) was used to measure the $\zeta$-potential.

\section{Synthesis of the silica Janus nanosheets}

BDMPS $(20 \mathrm{mg})$ in toluene $(1 \mathrm{~mL})$ was added to a dispersion of $\mathrm{CaCO}_{3}(1 \mathrm{~g})$ in toluene $(12 \mathrm{~mL})$. The mixture was stirred at $400 \mathrm{rpm}$ for $24 \mathrm{~h}$ at room temperature. The mixture was centrifuged and washed with toluene three times, then dried under vacuum. A SAM of BDMPS was formed on the particle surface.

$\mathrm{CaCO}_{3} @ \mathrm{SAM}$ composite particles (1.0 g) were dispersed in ethanol $(15 \mathrm{~mL})$. After $30 \mathrm{~min}$, aqueous ammonia $(20 \mathrm{~mL})$ was added. The mixture was stirred at $400 \mathrm{rpm}$ for $24 \mathrm{~h}$ at room temperature, and then the system was centrifuged, washed with ethanol three times, and dried under vacuum. A silica Janus monolayer was formed on the particle surface and $\mathrm{CaCO}_{3} @$ nanomembrane composite particles were obtained. The silica nanosheets were obtained after using $1 \mathrm{M}$ aqueous $\mathrm{HCl}$ solution $(5 \mathrm{~mL})$ under ultrasonication to etch the $\mathrm{CaCO}_{3}$ particles.

\section{Synthesis of the amine/acid composite Janus nanosheets}

The $\mathrm{CaCO}_{3} @$ nanomembrane composite particles (1.0 g)

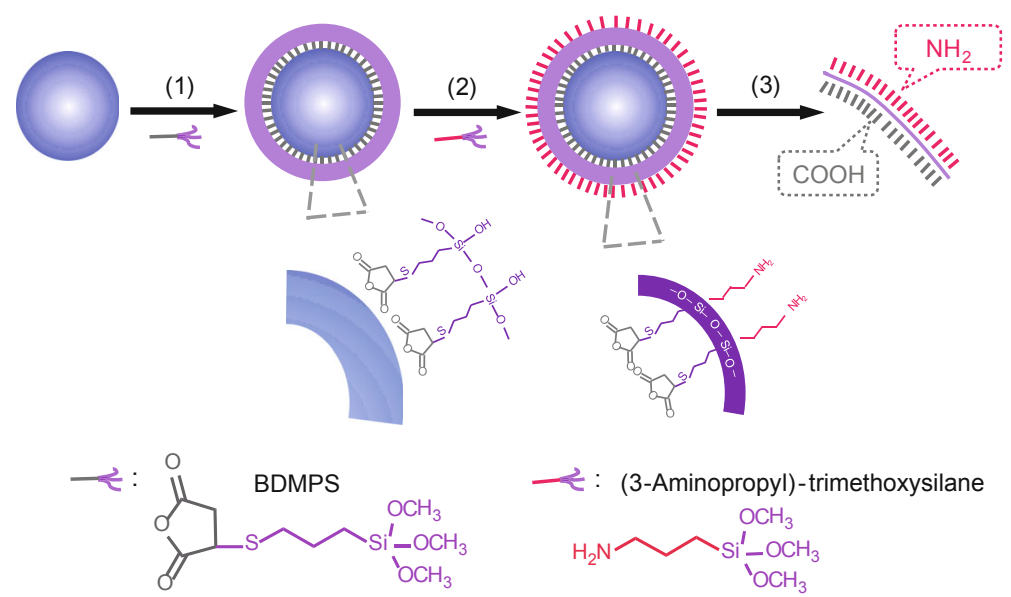

February 2015 | Vol.58 No.2
Scheme 1 Synthesis of the amine/acid composite Janus nanosheets. (1) Self-assembled monolayer after preferential adsorption of the amphiphilic silane BDMPS onto the template surface, and Janus silica nanomembrane formed from a surface sol-gel process; the $\mathrm{Si}-\mathrm{OH}$ groups are positioned at the exterior surface. (2) Janus silica nanomembrane after selective modification of the exterior silica surface with (3-aminopropyl)trimethoxysilane. (3) Amine/acid composite Janus nanosheets after acid etching of the template under ultrasonication. 
were dispersed in toluene (15 mL) and (3-aminopropyl) trimethoxysilane $(60 \mu \mathrm{L})$ was added. The mixture was refluxed at $80^{\circ} \mathrm{C}$ for $8 \mathrm{~h}$. After cooling to room temperature, the dispersion was centrifuged, washed with toluene three times, and dried under vacuum. $\mathrm{CaCO}_{3} @$ amine/maleic anhydride nanomembrane composite particles were obtained.

At $50^{\circ} \mathrm{C}, \mathrm{CaCO}_{3} @$ nanomembrane composite particles (500 mg) were added to a $1 \mathrm{M}$ aqueous $\mathrm{HCl}$ solution $(5 \mathrm{~mL}$ ) under ultrasonication. After cooling to room temperature, the dispersion was centrifuged and washed with water to give the amine/acid composite Janus nanosheets.

\section{Synthesis of the chitosan-capped $\mathrm{Fe}_{3} \mathrm{O}_{4}$ nanoparticles}

The chitosan-capped $\mathrm{Fe}_{3} \mathrm{O}_{4}$ nanoparticles were synthesized according to a literature procedure [19]. An aqueous solution $(44.0 \mathrm{~mL})$ containing $\mathrm{FeCl}_{3} \cdot 6 \mathrm{H}_{2} \mathrm{O}(0.70 \mathrm{~g})$ and $\mathrm{FeCl}_{2} \cdot 4 \mathrm{H}_{2} \mathrm{O}(0.30 \mathrm{~g})$ was injected into an aqueous solution of acetic acid [ $200 \mathrm{~mL}, 0.25 \%(\mathrm{v} / \mathrm{v})$ ]. Chitosan was added at $2.50 \mathrm{~g} / \mathrm{L}$ under nitrogen atmosphere. After stirring at $40^{\circ} \mathrm{C}$ for $1 \mathrm{~h}$, ammonium hydroxide $(20.0 \mathrm{~mL})$ was dropped into the solution under vigorous stirring over $30 \mathrm{~min}$. Chitosan-capped $\mathrm{Fe}_{3} \mathrm{O}_{4}$ nanoparticles were collected using a magnet and washed with water.

Synthesis of the trisodium-citrate-capped Au nanoparticles Au nanoparticles were prepared by the Frens method [20]. An aqueous solution of $\mathrm{HAuCl}_{4}\left(100 \mathrm{~mL}, 0.1 \mathrm{mg} \mathrm{mL}^{-1}\right)$ was refluxed under stirring and an aqueous solution of trisodium citrate $\left(2 \mathrm{~mL}, 10 \mathrm{mg} \mathrm{mL}^{-1}\right)$ was added. A dispersion of Au nanoparticles with an average diameter of $15 \mathrm{~nm}$ was obtained after $40 \mathrm{~min}$.

\section{Labeling the Janus nanosheets with the Au nanoparticles}

The Janus nanosheets $(1.0 \mathrm{mg})$ were dispersed in water. A small amount of the trisodium-citrate-capped Au nanoparticles was added to the dispersion. The as-formed mixture was stirred for $12 \mathrm{~h}$ to selectively label the nanosheets. The nanosheets were centrifuged and washed with water.

\section{pH responsive dispersion of the Janus nanosheets}

After the Janus nanosheets were dispersed in aqueous solution under ultrasonication for $5 \mathrm{~min}$ at various $\mathrm{pH}$ levels, the dispersions were left to stand for observation.

\section{RESULTS AND DISCUSSION}

A silica Janus nanomembrane was formed in a sol-gel process involving the self-assembly of a BDMPS monolayer on the $\mathrm{CaCO}_{3}$ particle surface (Fig. 1a). The nanomembrane was partially peeled from the particle surface under sonication. After the $\mathrm{CaCO}_{3}$ particles were etched with acid under ultrasonication, the silica nanomembrane was broken into
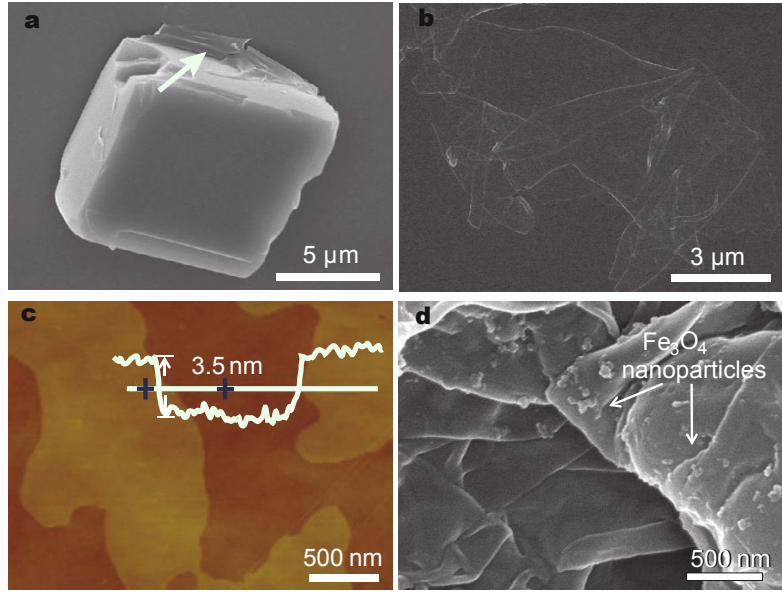

Figure 1 (a) Silica Janus nanomembrane after a sol-gel process involving the self-assembly of a BDMPS monolayer on the $\mathrm{CaCO}_{3}$ particle surface. (b) Silica Janus nanosheets after acid etching of the $\mathrm{CaCO}_{3}$ particle under ultrasonication. (c) AFM image of the silica Janus nanosheets, which have a thickness of $3.5 \pm 0.2 \mathrm{~nm}$. (d) Silica Janus nanosheets after positively charged $\mathrm{Fe}_{3} \mathrm{O}_{4}$ nanoparticles are preferentially adsorbed onto the carboxyl group terminated side.

small pieces and the silica Janus nanosheets were formed (Fig. 1b). At the same time, the acid anhydride group was hydrolyzed to give a carboxyl group. Both sides of the silica Janus nanosheets are smooth, and the sheets are flexible and easily wrinkled. The average thickness is $3.5 \pm 0.2 \mathrm{~nm}$ (Fig. 1c), which is close to the calculated value of $3.3 \mathrm{~nm}$ [18]. Positively charged chitosan-capped $\mathrm{Fe}_{3} \mathrm{O}_{4}$ nanoparticles were used to selectively label the carboxyl group terminated side. As a result, this side became coarse, whereas the other side remained smooth (Fig. 1d). In addition, the Janus nanosheets could be manipulated under a magnetic field.

Prior to etching the $\mathrm{CaCO}_{3}$, that is, while the anhydride group terminated interior surface is protected, desired groups can be selectively conjugated onto the exterior silica surface of the Janus nanomembrane using silanes. As an example, (3-aminopropyl)trimethoxysilane was used. The corresponding amine/acid composite Janus nanosheets were obtained after etching the $\mathrm{CaCO}_{3}$ under ultrasonication (Fig. 2a). These nanosheets are $3.9 \pm 0.2 \mathrm{~nm}$ thick (Fig. 2b), which is slightly thicker than the silica Janus nanosheets. This increase in thickness originates from the conjugation of (3-aminopropyl)trimethoxysilane on one side of the nanosheet. When negatively charged $\mathrm{Au}$ nanoparticles were selectively adsorbed onto the amine group terminated side of the amine/acid nanosheet, this side became coarse whereas the acid group terminated side remained smooth (Fig. 2c). In comparison, both sides of the silica Janus nanosheets remained smooth after treatment with Au nanoparticles. No Au nanoparticles were found on 

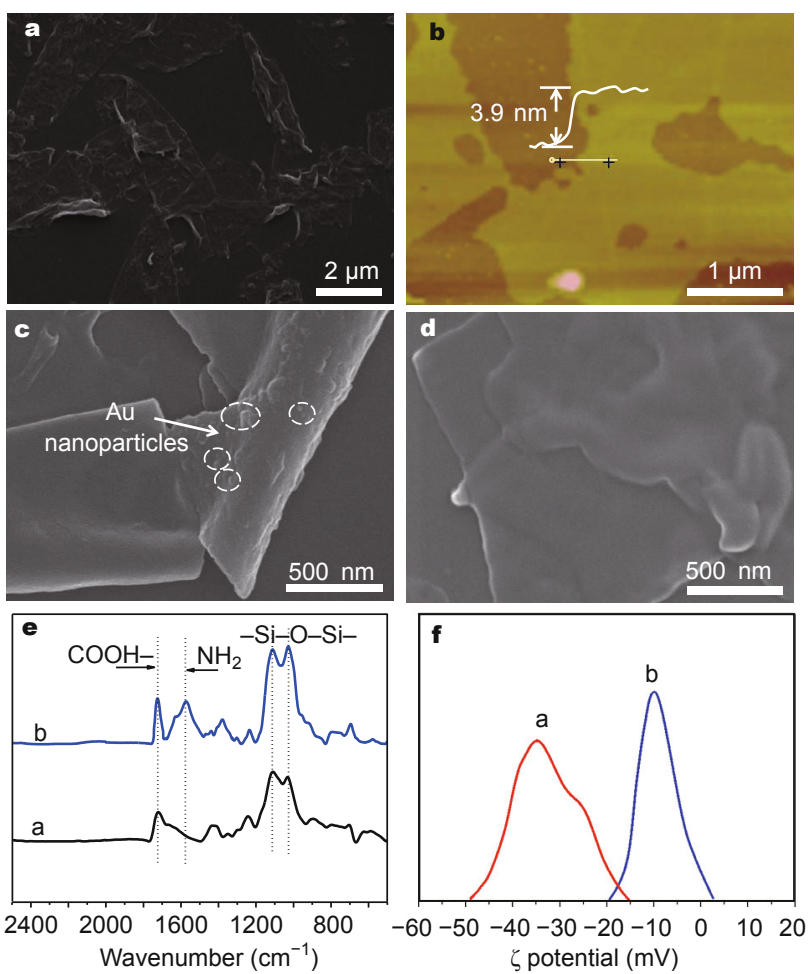

Figure 2 (a) Amine/acid composite Janus nanosheets after etching of the $\mathrm{CaCO}_{3}$ particles under ultrasonication. (b) AFM image of the Janus nanosheets with a thickness of $3.9 \pm 0.2 \mathrm{~nm}$. Negatively-charged-Aunanoparticle-labeled amine/acid composite Janus nanosheets (c) and silica Janus nanosheets (d). All samples were obtained after drying the dispersion in ethanol. (e) FTIR spectra of silica Janus nanosheets (curve a) and amine/acid composite nanosheets (curve b). (f) $\zeta$-potential of the silica Janus nanosheets (curve a) and the amine/acid composite nanosheets (curve b). All measurements were performed at $\mathrm{pH}$ 3.9.

the silica Janus nanosheets (Fig. 2d). In the FTIR spectrum of the silica Janus nanosheets (curve a, Fig. 2e), the peaks at $1024 \mathrm{~cm}^{-1}$ and $1098 \mathrm{~cm}^{-1}$ are assigned to the $-\mathrm{Si}-\mathrm{O}-\mathrm{Si}-$ of the silica. The peak at $1718 \mathrm{~cm}^{-1}$ is assigned to $-\mathrm{COOH}$. The new peak at $1568 \mathrm{~cm}^{-1}$ is assigned to the $-\mathrm{NH}_{2}$ of the amine/acid composite Janus nanosheets (curve b, Fig. 2e). The $\zeta$-potential of the silica Janus nanosheets is $-32.2 \mathrm{mV}$ and is attributed to the negatively charged carboxyl groups. After modification, the $\mathrm{Si}-\mathrm{OH}$ group is terminated with an amine group, and this can partially neutralize the carboxyl group. The $\zeta$-potential of the amine/acid composite Janus nanosheets is thus considerably reduced [-7.6 $\mathrm{mV}$ (Fig. 2f)].

The composite nanosheets possess two kinds of $\mathrm{pH}$-responsive groups, amine and carboxyl, which are located on opposite sides of the sheet. We were interested in systematically characterizing the dispersion behavior of the composite Janus nanosheets in aqueous solution at various $\mathrm{pH}$ levels (Fig. 3a). At low $(<4)$ or high $(>10) \mathrm{pH}$ levels, the nanosheets were well dispersed in aqueous solution. At in-
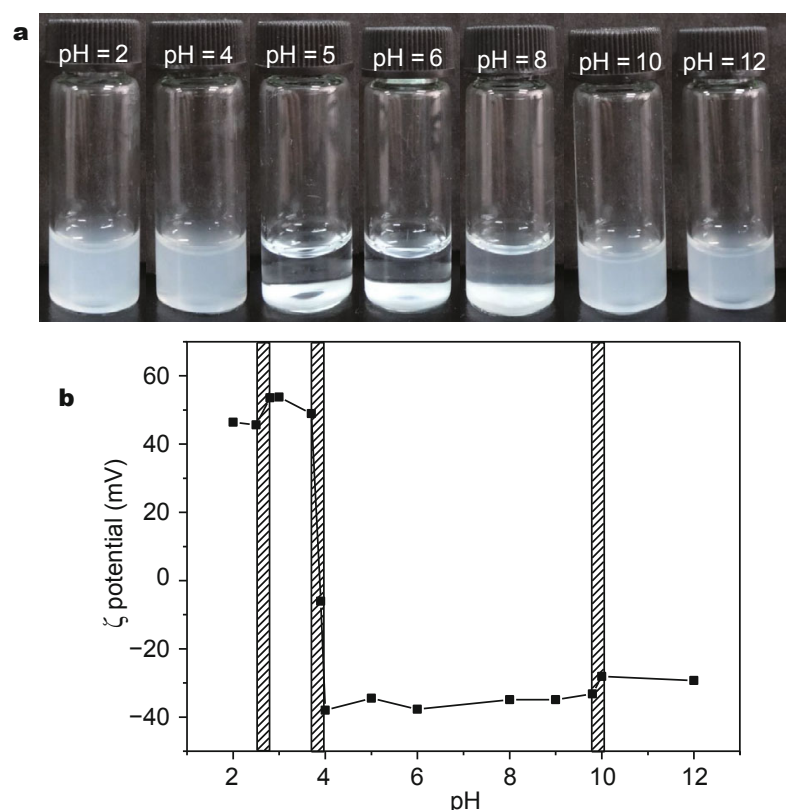

Figure 3 (a) Amine/acid composite Janus nanosheet dispersions in aqueous solution at various $\mathrm{pH}$ levels. (b) $\zeta$-potential dependence of the amine/acid composite Janus nanosheet dispersion on $\mathrm{pH}$.

termediate $\mathrm{pH}$ levels between $\mathrm{pH} 5$ and 8 , the nanosheets precipitated from aqueous solution. The top phase became transparent, which implied that all the nanosheets were present in the bottom phase. On adjusting the $\mathrm{pH}$ beyond the $\mathrm{pH} 5-8$ window and after ultrasonication, the aggregates became redispersible in aqueous solution. The $\zeta$-potential dependence of the dispersions on $\mathrm{pH}$ was monitored (Fig. 3b). The carboxyl group of the acid anhydride derivative exhibits two dissociations [21]. Between $\mathrm{pH} 2.5$ and 2.8 , the $\zeta$-potential changes abruptly but remains at a very high level above $40 \mathrm{mV}$. This corresponds to the positive charge of the protonated amine group, but the carboxyl group is not significantly dissociated at these $\mathrm{pH}$ values. A subtle increase in $\mathrm{pH}$ from 3.7 to 4.0 leads to a dramatic decrease in the $\zeta$-potential from $\sim 40 \mathrm{mV}$ to $-39.2 \mathrm{mV}$. The $\zeta$-potential is preserved at this plateau level with any further changes in $\mathrm{pH}$. This change arises from the major dissociation of the carboxyl group. Another abrupt increase in the $\zeta$-potential is observed on subtly increasing the $\mathrm{pH}$ from $\mathrm{pH} 9.8$ to 10.0 ; this corresponds to the dissociation of the amine group on the other side of the nanosheets. At $\mathrm{pH}$ values above 4.0, the carboxyl group is dissociated and becomes negatively charged. At $\mathrm{pH}$ values below 9.8, the amine group is protonated and becomes positively charged. The composite nanosheets are Janus materials with amphiphilic performance both below $\mathrm{pH} 4.0$ and above $\mathrm{pH} 9.8$. At $\mathrm{pH}$ values between $\mathrm{pH} 4.0$ and $\mathrm{pH} 9.8$, both the amine and carboxyl groups are highly charged but have opposite 
polarities. The nanosheets can form heavy aggregates because of the strong electrostatic interaction between the two groups.

After ambient drying of the aqueous dispersion at $\mathrm{pH}$ 2 , the nanosheets were folded and well distinguished (Fig. 4a). At this $\mathrm{pH}$, it is rational that the acid group terminated sides are stacked inwardly face-to-face with the protonated amine group exposed to the aqueous phase. We expected that a bilayered structure would form among the Janus nanosheets [8]. Surprisingly, individual nanosheets were self-folded into a wrinkled structure. This finding is similar to that of our previous study on the encapsulation of paraffin using single alkyl/acid Janus nanosheets [18]. The wrinkling is related to the flexibility of the nanosheets. At $\mathrm{pH}$ 6, the carboxyl group is dissociated and becomes negatively charged. Because of the opposite electrostatic interaction between the charged carboxyl and amine groups, the nanosheets should form a face-to-back superstructure and quickly precipitate from aqueous solution (Fig. 4b). To further clarify the structure of these superstructures in aqueous solution, the dispersion was cryo-dried. At $\mathrm{pH}$ 6, the nanosheets were stacked to form a multilayered superstructure due to the alternating opposite charge interaction (Fig. 4c). A magnified SEM image reveals local wrinkling of the nanosheets, implying they are rather flexible (Fig. $4 \mathrm{~d})$. At $\mathrm{pH} 12$, the morphology of the superstructure is similar to the structure at $\mathrm{pH} 2$ because the nanosheets are Janus materials.

\section{CONCLUSIONS}

In summary, we have proposed a facile method to fabricate amphiphilic amine/acid composite Janus nanosheets. Firstly, a silica Janus nanosheet with carboxyl group onto one
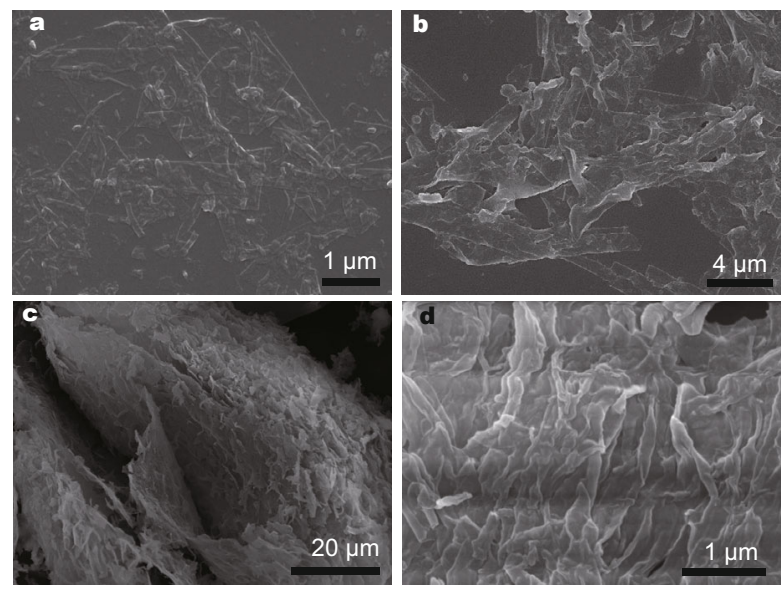

Figure 4 SEM images of the amine/acid composite nanosheets after ambient drying of the aqueous dispersion at $\mathrm{pH} 2$ (a) and $\mathrm{pH} 6$ (b). (c, d) SEM images of the nanosheets after cryo-drying of the aqueous dispersion at $\mathrm{pH} 6$. side is obtained by sol-gel process of a self-assembly monolayer of an amphiphilic silane BDMPS onto a solid particle template surface by a coordination interaction. The amine group is further terminated onto the other side of the carboxyl group teminated Janus nanosheet. Both the acid and amine groups are distinctly compartmentalized onto the two sides, respectively. Both sides can be $\mathrm{pH}$ responsive in a separate way. The nanosheets are dually $\mathrm{pH}$ responsive. The nanosheets are well-dispersible at both low and high $\mathrm{pH}$ levels. At intermediate $\mathrm{pH}$, a multilayered superstructure is formed by face-to-back stacking due to interaction between the opposite electrostatic charges on the opposite sides of the nanosheets. The method is general to fabricate other functional Janus nanosheets. Thanks to their stimuliresponsive performance, these flexible Janus nanosheets may be potential in controlled loading and release, and heterogeneous catalysis.

Received 22 December 2014; accepted 21 January 2015; published online 10 February 2015

1 de Gennes PG. Soft matter. Rev Mod Phys, 1992, 64: 645-648

$2 \mathrm{Hu}$ J, Zhou SX, Sun YY, et al. Fabrication, properties and applications of Janus particles. Chem Soc Rev, 2012, 41: 4356-4378

3 Jiang S, Granick S, Schneider HJ. Janus Particle Synthesis, Self-Assembly and Applications. London: Royal Society of Chemistry, 2012

4 Walther A, Müller AHE. Janus particles: synthesis, self-assembly, physical properties, and applications. Chem Rev, 2013, 113: 51945261

5 Zhang HB, Hao R, Jackson JK, et al. Janus ultrathin film from multilevel self-assembly at air-water interfaces. Chem Commun, 2014, 50: 14843-14846

6 Liang FX, Zhang CL, Yang ZZ. Rational design and synthesis of Janus composites. Adv Mater, 2014, 26: 6944-6949

7 Nonomura Y, Komura S, Tsujii K. Adsorption of disk-shaped Janus beads at liquid-liquid interfaces. Langmuir, 2004, 20: 11821-11823

8 Liang FX, Shen K, Qu XZ, et al. Inorganic Janus nanosheets. Angew Chem Int Ed, 2011, 50: 2379-2382

9 Walther A, André X, Drechsler M, et al. Janus discs. J Am Chem Soc, 2007, 129: 6187-6198

10 Wang Y, Zhong WHK, Ji JY, et al. Blossoming of nanosheet structures via a disturbed self-assembly. Nano Lett, 2014, 14: 3474-3480

11 Qi H, Wang WD, Li CY. Janus polymer single crystal nanosheet via evaporative crystallization. ACS Macro Lett, 2014, 3: 675-678

12 Dorvee JR, Derfus AM, Bhatia SN, et al. Manipulation of liquid droplets using amphiphilic, magnetic one-dimensional photonic crystal chaperones. Nat Mater, 2004, 3: 896-899

13 Kirillova A, Stoychev G, Ionov L, et al. Platelet Janus particles with hairy polymer shells for multifunctional materials. ACS Appl Mater Interfaces, 2014, 6: 13106-13114

14 Liang FX, Liu JG, Zhang CL, et al. Janus hollow spheres by emulsion interfacial self-assembled sol-gel process. Chem Commun, 2011, 47: 1231-1233

15 Yang HL, Liang FX, Wang X, et al. Responsive Janus composite nanosheets. Macromolecules, 2013, 46: 2754-2759

16 Zheng ZK, Nottbohm CT, Turchanin A. Janus nanomembranes: a generic platform for chemistry in two dimensions. Angew Chem Int Ed, 2010, 49: 8493-8497

17 Zhang LM, Yu JW, Yang MM, et al. Janus graphene from asymmetric two-dimensional chemistry. Nat Commun, 2012, 4: 1443-1149 
18 Liu YJ, Liang FX, Wang Q, et al. Flexible responsive Janus nanosheets. Chem Commun, doi: 10.1039/C4CC08420A

19 Wang JZ, Zhao GH, Li YF, et al. Reversible immobilization of glucoamylase onto magnetic chitosan nanocarriers. Appl Microbiol Biot, 2012, 97: 681-692

20 Frens, G. Controlled nucleation for the regulation of the particle size in monodisperse gold suspensions. Nat Phys Sci, 1973, 241: $20-22$

21 Tu FQ, Lee D. Shape changing and amphiphilicity reversing Janus particles with $\mathrm{pH}$-responsive surfactant properties. J Am Chem Soc, 2014, 136: 9999-10006
Acknowledgements This work was supported by Ministry of Science and Technology of China (2012CB933200) and the National Natural Science Foundation of China (51233007 and 51173191).

Author Ccontributions Yang Z proposed the project; Yang Z and Liang F designed the work and wrote the manuscript; Liu Y synthesized the materials and measured the properties. Wang $\mathrm{Q}$ and $\mathrm{Qu} \mathrm{X}$ participated in the analysis of the experimental data and discussion of the results.

Conflict of interest The authors declare that they have no conflicts of interest.

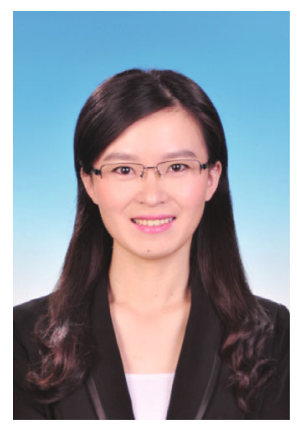

Yijiang Liu was born in 1984. She received her MSc degree in polymer chemistry and physics from Xiangtan University in 2009. She worked as a lecturer at Hunan University of Humanities, Science and Technology from 2010 to 2012. In 2012, she joined Yang's group to pursue her PhD degree. Her research concerns the synthesis and performance of 2D Janus nanosheets and the synthesis of polymer Janus materials by living polymerization.

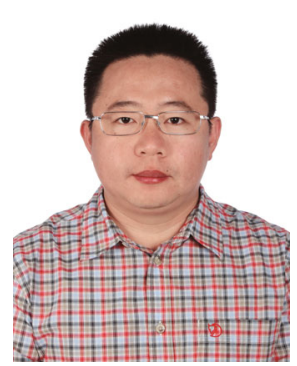

Fuxin Liang was born in 1981. He received his MSc degree in inorganic chemistry from Liaoning University in 2007. After he received his PhD degree in polymer physics and chemistry from the Institute of Chemistry, Chinese Academy of Sciences (ICCAS) in 2010, he joined Yang's group and he is currently an associate professor. His research interests include the design, synthesis and application of Janus materials and phase-change materials.

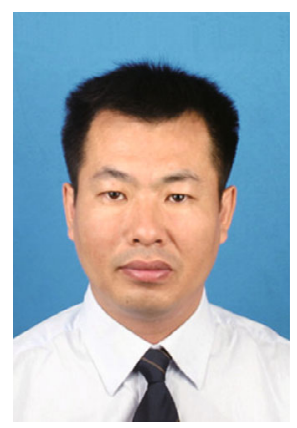

Zhenzhong Yang was born in 1968. He received his BSc from Tsinghua University (1991), MSc from Jilin University (1994) and PhD from the Institute of Chemistry, Chinese Academy of Sciences (1997). He then did postdoctoral research at the BASF polymer central laboratory in Ludwigshafen (1997-1998). He is a professor and director of the Laboratory of Polymer Physics and Chemistry at ICCAS. His research interests include polymer composites with precisely tunable macroscopic morphology, and the microstructure/spatial distribution of their components. The composites include polymer-based composite hollow spheres, functional capsules and mesoporous membranes. He is especially interested in asymmetric Janus composites and their practical applications.

中文摘要 含酸䣲两亲硅烷偶联剂在颗粒表面吸附形成自组装单分子膜, 可通过溶胶-凝胶法制备二氧化硅Janus纳米片. 本文通过对 二氧化硅Janus纳米片选择改性在一侧偶联氨基, 制备了羧基/氨基复合的Janus纳米片. 纳米片两侧的羧基与氨基具有不同的 $\mathrm{pH}$ 值响应 性, 表现出相应的荷电性质及润湿性. 二者共同作用, 引起纳米片特殊的分散和聚集行为. 在较低或较高pH值水平, 纳米片表现为Janus 特性, 分散良好. 在中间 $\mathrm{pH}$ 值范围, 纳米片两侧羧基、氨基相反电荷相互吸引导致层层组装聚集并沉淀. 